MATHEMATICS OF COMPUTATION

Volume 76, Number 260, October 2007, Pages 1787-1800

S 0025-5718(07)01993-X

Article electronically published on May 3, 2007

\title{
SHARP ESTIMATES FOR FINITE ELEMENT APPROXIMATIONS TO ELLIPTIC PROBLEMS WITH NEUMANN BOUNDARY DATA OF LOW REGULARITY
}

\author{
AARON SOLO
}

\begin{abstract}
Consider a second order homogeneous elliptic problem with smooth coefficients, $A u=0$, on a smooth domain, $\Omega$, but with Neumann boundary data of low regularity. Interior maximum norm error estimates are given for $C^{0}$ finite element approximations to this problem. When the Neumann data is not in $L^{1}(\partial \Omega)$, these local estimates are not of optimal order but are nevertheless shown to be sharp. A method for ameliorating this suboptimality by preliminary smoothing of the boundary data is given. Numerical examples illustrate the findings.
\end{abstract}

\section{INTRODUCTION}

Let $\Omega$ be a bounded domain with a smooth boundary in $R^{N}$ and let $A$ be a symmetric uniformly elliptic second order operator with smooth coefficients such that the associated bilinear form is coercive in the sense that $A(v, v)>c\|v\|_{H^{1}}^{2}$ for all $v \in H^{1}$. We are interested in the finite element approximation of the solution to the problem with a natural boundary condition,

$$
\begin{gathered}
A u=0 \quad \text { in } \Omega, \\
u_{\nu_{A}}=f \quad \text { on } \quad \partial \Omega,
\end{gathered}
$$

where $u_{\nu_{A}}$ is the co-normal derivative of $u$, and the boundary data, $f$, in general, will have low regularity. The behavior of the finite element method on this type of problem is relevant to, for instance, problems in elasticity involving concentrated loads and problems where the specified data has high frequency oscillations. We give an example of this second application at the end of Section 3.

The variational formulation of this problem is as follows. Find $u \in H^{1}(\Omega)$ such that

$$
A(u, v)=\langle f, v\rangle_{\partial \Omega}, \quad \forall v \in H^{1}(\Omega)
$$

where $A(\cdot, \cdot)$ is the bilinear form associated with $A$ and $\langle\cdot, \cdot\rangle$, here and below, denotes the pairing of a linear space with its dual. By standard trace inequalities, this formulation is well posed for $f \in H^{-1 / 2}(\partial \Omega)$. It is, however, possible to pose the problem for $f \in W_{p}^{-k}$ for any $k$ and $p$, and we will discuss this further in

Received by the editor April 11, 2006 and, in revised form, August 2, 2006.

2000 Mathematics Subject Classification. Primary 65N30.

Key words and phrases. Finite element methods, boundary value problems, low regularity data.

(C)2007 American Mathematical Society

Reverts to public domain 28 years from publication 
Section 2. The important point is that, in either case, the solution, $\mathrm{u}$, has regularity $\|u\|_{H^{3 / 2-k}(\Omega)} \leq C\|f\|_{H^{-k}(\partial \Omega)}$.

Now let $S_{r}^{h}$ be some finite element subspace of $H^{1}(\Omega)$ and consider the approximation to $u, u_{h}$, defined by

$$
A\left(u_{h}, v\right)=\langle f, v\rangle_{\partial \Omega}, \forall v \in S_{r}^{h} .
$$

If $S_{r}^{h}$ is a space of continuous piecewise polynomials of degree $r-1$ on a mesh of size $h$, then by approximation theory we might hope for the error, $u-u_{h}$, to satisfy an estimate of the form

$$
\left\|u-u_{h}\right\|_{L^{2}(\Omega)} \leq C h^{3 / 2-k}\|u\|_{H^{3 / 2-k}(\Omega)} \leq C h^{3 / 2-k}\|f\|_{H^{-k}(\partial \Omega)}, \quad \text { for } 3 / 2-k \leq r .
$$

Estimates analogous to this for the Dirichlet problem have been studied extensively, and results have been obtained for $u$ having regularity as low as $H^{1 / 2}(\Omega)$; see $[6$, [9] and [8. We note that this is nearly as low as one can go and still hope for any convergence whatsoever, at least over the whole domain.

However, returning for a moment to the continuous problem, we recall that, although $u$ may have very low global regularity, for any subdomain $B \subset \subset \Omega$ we have $u \in C^{\infty}(B)$. It is therefore conceivable that $u_{h}$ might converge at a much higher rate on such a subdomain, and even that $u_{h}$ might converge on a subdomain while failing to converge globally. This is the idea that we will pursue in this paper.

The idea that it is possible to obtain higher rates of convergence on subdomains where the solution is smoother is not a new one. Localization estimates roughly of the form,

$$
\left\|u-u_{h}\right\|_{X(B)} \leq C\left\{\min _{\chi \in S_{r}^{h}}\|u-\chi\|_{X\left(B^{\prime}\right)}+\left\|u-u_{h}\right\|_{H^{-s}(\Omega)}\right\},
$$

where $B \subset B^{\prime}, X(B)$ can be either $H^{1}(B)$ or $L^{\infty}(B)$ and $s$ is any nonnegative number, were first obtained by Nitsche and Schatz in $[12$ and Schatz and Wahlbin in 13 and are reviewed in [15].

Examining these estimates, we see that one approach to the problem at hand would be to use the local approximation properties of $S_{r}^{h}$ to bound the first term on the right, and then to find a way to bound the second term on the right, which is global, but is also in a weaker norm. This is the approach taken in a recent paper by Babuška and Nistor [3, where the authors show that it is possible to obtain convergence on an interior domain for problems with very low global regularity by using the generalized finite element method, a method which differs from the standard finite element method in several respects, including that the subspaces used are required to have higher order global regularity than that of standard $C^{0}$ finite element subspaces. In this context, they are able to prove a bound of the form

$$
\left\|u-u_{h}\right\|_{H^{1}(B)} \leq C h^{r-1 / 2-k}\|u\|_{H^{3 / 2-k}(\Omega)} \leq C h^{r-1 / 2-k}\|f\|_{H^{-k}(\partial \Omega)}
$$

where $r$ is the local approximation degree of the subspace $S_{r}^{h}$ and $k \geq 1 / 2$.

There are, however, two substantial limitations to the results obtained in [3]. First, although the estimates in that paper are a clear improvement over the best possible global results, for $k>1 / 2$ they are still not of optimal order. Second, and most importantly, these results do not apply to standard finite element methods, 
since the method of proof relies fundamentally on the high regularity of the generalized finite element subspaces to obtain bounds for the global negative norm term in (44).

This requirement that the subspaces have higher regularity for problems where the boundary data has lower regularity may seem unavoidable; for the standard $C^{0}$ finite element method, $\left.S^{h}\right|_{\partial \Omega} \notin W_{\infty}^{k}$ for $k>1$, so problem (3) is only well posed if $f \in W_{1}^{-k}$ for $k \leq 1$. However, for many problems of practical interest, the data falls within this range of regularity. Furthermore, we will see below that even outside of this range it is possible to avoid using the generalized finite element method by instead smoothing the data.

In this paper we therefore aim to extend the results in [3] to apply to standard finite element methods and to include sharp $L^{\infty}$ estimates. Since the method of proof in [3] requires the high regularity of the generalized finite element subspaces, we must take a somewhat different approach here, although the same fundamental localization estimates from equation (4) remain at the core of our method. In brief, we start with the error representation

$$
u(x)-u_{h}(x)=\left\langle G^{x}-G_{h}^{x}, f\right\rangle_{\partial \Omega}
$$

where $x$ is an arbitrary point in $B \subset \subset \Omega$ and $G^{x}$ and $G_{h}^{x}$ are the continuous and discrete Green's functions. Then, following [13] and [15], we use a precise version of (44) to derive pointwise estimates for $\left|G^{x}-G_{h}^{x}\right|$. The end result is an estimate of the form

$$
\left\|u-u_{h}\right\|_{L^{\infty}(B)} \leq C(d) h^{r-k}\|f\|_{W_{1}^{-k}}
$$

where $d=d(B, \partial \Omega), k=0$ or 1 , and $r$ is the approximation degree of $S_{r}^{h}$. For $k=0$ this gives the optimal rate of convergence. For $k=1$, on the other hand, this estimate is no longer locally optimal, but we will show that it is, nevertheless, sharp. We will also give a sharp estimate for the dependence of $C(d)$ on $d$.

For purposes of comparison to global results and the results in [3], we take the example of $f \in L^{1}(\partial \Omega)$ and $\Omega \subset R^{2}$. In this case the most we can say about $u$ is that $\|u\|_{H^{1-\epsilon}(\Omega)} \leq C\|f\|_{H^{-1 / 2-\epsilon}(\partial \Omega)} \leq C\|f\|_{L^{1}(\partial \Omega)}$. Thus, the best possible global estimates are $O\left(h^{1-\epsilon}\right)$ in $L^{2}$, regardless of the approximation degree of the subspace. If one is willing to work with the generalized finite element method, then [3] improves on this, giving $O\left(h^{r-1-\epsilon}\right)$ convergence in either $H^{1}(B)$ or $L^{2}(B)$. Our estimate, on the other hand, applies to the standard finite element method and gives optimal $O\left(h^{r}\right)$ convergence in $L^{\infty}(B)$.

Since problem (3) is not well posed for for the standard finite element method for general $f \in W_{1}^{-k}$ if $k>1$, estimate (5D) cannot be extended directly to the case $k>1$. However, as noted above, it is possible to work around this difficulty by pre-smoothing the boundary data, and we discuss this in detail in Section 3. As a consequence of the sharpness of our results we are able to give a precise description of when this is beneficial.

The remainder of this paper is organized as follows. Section 2 contains the precise formulation and proof of our error estimates, as well as the proof of the sharpness of these estimates. In Section 3 we discuss a method of pre-smoothing the data and prove rates of convergence for different choices of smoothing kernels. Finally, in Section 4 we give some numerical results. 


\section{ERROR BOUNDS AND SHARPNESS}

Before we can state our results, we must take a moment to establish our notation and assumptions and to define the solution to problem (1) in the case of very weak boundary data.

We begin by defining the negative order Sobolev spaces. For $B$ any subdomain of $\Omega, s>0$, and $1<q<\infty$ we define $W_{q}^{-s}(B)$ to be the space of all linear functionals, $f$, on $C_{0}^{\infty}(B)$ such that

$$
\sup _{\phi \in C_{0}^{\infty}(B)} \frac{\langle f, \phi\rangle}{\|\phi\|_{W_{p}^{s}(B)}}<\infty
$$

where $p=\frac{q}{q-1}$, and we equip this space with the obvious norm. For $q=2$, we denote this space by $H^{-s}(B)$, and note that, using density, any element of $H^{-s}(B)$ can be extended to an operator on $H_{0}^{s}(B)$ with the same norm. For $1<q<\infty$ we define the negative order spaces on $\partial \Omega$ analogously. The spaces $W_{1}^{-k}(\partial \Omega)$ play a central role in our estimates. For fixed $k$, we define this to be the space of all linear functionals on $W_{\infty}^{k}(\partial \Omega)$ such that

$$
\sup _{\phi \in W_{\infty}^{k}(\partial \Omega)} \frac{\langle f, \phi\rangle}{\|\phi\|_{W_{\infty}^{k}(\partial \Omega)}}<\infty,
$$

again equipped with the obvious norm. This is a somewhat nonstandard definition, but it is the most natural one for our purposes. (A more standard definition would use $\phi \in C^{\infty}$.)

We can now give the definition of the solution to problem (1) for weak boundary data. Given $k \geq 3 / 2$ and $f \in H^{-k}(\partial \Omega)$, we define our solution, $u$, as follows. Given $\phi \in H_{0}^{k-3 / 2}(\Omega)$, we let $w_{\phi}$ be the unique solution of

$$
A\left(w_{\phi}, v\right)=(\phi, v), \quad \forall v \in H^{1}(\Omega)
$$

and note that $\left.w_{\phi}\right|_{\partial \Omega} \in H^{k}(\partial \Omega)$, so it makes sense to define $u$ as the element of $H^{3 / 2-k}(\Omega)$ such that

$$
\langle u, \phi\rangle=\left\langle f, w_{\phi}\right\rangle_{\partial \Omega}, \quad \forall \phi \in H_{0}^{k-3 / 2}(\Omega) .
$$

One can check that, in the case of smooth data, this definition coincides with the definitions given in equations (11) and (2). For instance, if $u$ solves (2) with data in $H^{-1 / 2}$, then

$$
(\phi, u)=A\left(w_{\phi}, u\right)=A\left(u, w_{\phi}\right)=\left\langle f, w_{\phi}\right\rangle_{\partial \Omega}, \quad \forall \phi \in H^{-1} .
$$

We note that the solution operator is bounded from $H^{-k}(\partial \Omega)$ to $H^{3 / 2-k}(\Omega)$ for $k \leq 1 / 2$ and for $k \geq 3 / 2$. For intermediate values we obtain the same result by interpolation. Since $W_{q}^{-k}(\partial \Omega) \subset H^{-l}(\partial \Omega)$ for $l$ large enough, this immediately gives us a definition for the solution to problem (1) for data in any $W_{q}^{-k}$ space. In what follows, it will be important that the operator is also bounded from $W_{q}^{-k}(\partial \Omega)$ to $L^{\infty}(B)$, for $B \subset \subset \Omega$. To see this, note that since $u$ satisfies a homogeneous elliptic equation on $\Omega$, we have $\|u\|_{C^{m}(B)} \leq C(B)\|u\|_{H^{-k}(\Omega)}$ for any $m$ and any $k$. Combining this with the above gives the result. For more details on solutions to problems with weak boundary data, we refer the reader to [11, Chapter 2 and also to $[2]$.

Now moving on to the discrete problem, we make the following assumptions on the finite element subspaces. First, we assume that for every $0<h<1$ we have a triangulation of $\Omega,\left\{\tau_{i}^{h}\right\}$, which fits the boundary exactly. In two dimensions this is 
easily accomplished by extending or contracting the elements abutting the boundary to form pie-shaped elements. In three dimensions the situation is more complicated since the procedure which works in two dimensions results in overlapping elements. One way to overcome this problem is by extending $\Omega$ by $O\left(h^{2}\right)$, triangulating the extended domain to form a polygonal domain, $\Omega_{h}$, such that $\Omega \subset \Omega_{h}$, and then restricting the triangulation to $\Omega$. For more on triangulating curved domains we refer the reader to 1] and 4. In addition to fitting the boundary exactly, we assume that the triangulations are globally quasi-uniform and shape regular. By this we mean that there are constants $c$ and $C$, independent of $h$, such that every $\tau_{i}^{h}$ can be inscribed with a ball of radius $>c h$ and circumscribed by a ball of radius $<C h$. Finally, we assume that $S_{r}^{h}$ is the space of continuous functions on $\Omega$ which are polynomials of degree $\leq r-1$ on each triangle, $\tau_{i}^{h}$.

From this structure, it follows that the subspaces, $S_{r}^{h}$, will satisfy the usual approximation and inverse assumptions, as well as the more esoteric trace inequality and super-approximation assumptions which are necessary for some of the results quoted in this paper. For more details on these matters we refer the reader to [13] and $[12$.

In all that follows $\Omega_{d}=\{x \in \Omega, d(x, \partial \Omega)>d\}, C_{d, r}=C \log (1 / d) d^{-N-r+2}$, where $N$ is the space dimension, and $\ell_{h, r}=\log (1 / h)^{p}$, where $p=1$ for $r=2$, and $p=0$ for $r>2$. We are now ready to state our results for low regularity boundary data without any preliminary smoothing.

Theorem 1. Under the preceding assumptions, and with $u_{h}$ as defined by equation (3), we have, for $k=0,1$,

$$
\left\|u-u_{h}\right\|_{L^{\infty}\left(\Omega_{d}\right)} \leq C_{d, r} \ell_{h, r} h^{r-k}\|f\|_{W_{1}^{-k}(\partial \Omega)} .
$$

Furthermore, modulo the factor $\ell_{h, r}$, these convergence rates are sharp.

2.1. Proof of Bounds. We first establish an error representation in terms of the discrete and continuous Green's functions. To this end, we begin by defining the discrete Green's function centered at $x \in \Omega, G_{h}^{x} \in S_{r}^{h}$, by the relationship,

$$
A\left(G_{h}^{x}, v\right)=v(x), \quad \forall v \in S_{r}^{h}
$$

and note that, since $A(\cdot, \cdot)$ is symmetric, for any $x, y \in \Omega$,

$$
G_{h}^{x}(y)=A\left(G_{h}^{y}, G_{h}^{x}\right)=G_{h}^{y}(x) .
$$

Now, combining the definitions of $G_{h}^{x}$ and $u_{h}$, we have

$$
u_{h}(x)=A\left(G_{h}^{x}, u_{h}\right)=\left\langle G_{h}^{x}, f\right\rangle_{\partial \Omega} .
$$

Note that the right hand side of this equation makes sense, since $\left.G_{h}^{x}\right|_{\partial \Omega} \in W_{\infty}^{1}(\partial \Omega)$ and we are assuming that $f \in W_{1}^{-k}$ for $k \leq 1$.

Turning to the continuous problem, if we let $G^{x}$ be the Green's function for the Neumann problem and if $u$ is the solution to problem (11) with smooth data, then we have the familiar formula

$$
u(x)=\left\langle G^{x}, f\right\rangle_{\partial \Omega},
$$

which is obtained using integration by parts and the fact that $G^{x}$ solves the problem

$$
\begin{aligned}
A G^{x} & =\delta^{x}, \\
G_{\nu_{A}}^{x} & =0 \text { on } \partial \Omega .
\end{aligned}
$$


We want to show that this formula remains valid for $f$ in $W_{p}^{k}$ for any $k$ and $1 \leq p \leq$ $\infty$. Note that $W_{p}^{k} \subseteq H^{-s}$ for $s$ large enough, so it suffices to prove the formula for $f \in H^{-s}$. For this purpose, given $f \in H^{-s}(\partial \Omega)$ we choose a sequence $f_{\epsilon} \in C^{\infty}(\partial \Omega)$ such that $\left\|f-f_{\epsilon}\right\|_{H^{-s}(\partial \Omega)} \leq \epsilon$. Then, letting $u_{\epsilon}$ solve the boundary value problem with data $f_{\epsilon}$, we obtain

$$
u(x)=\lim _{\epsilon \rightarrow 0} u_{\epsilon}(x)=\lim _{\epsilon \rightarrow 0}\left\langle G^{x}, f_{\epsilon}\right\rangle_{\partial \Omega}=\left\langle G^{x}, f\right\rangle_{\partial \Omega}
$$

where the first equality follows by the boundedness of the solution operator from $H^{-s}(\partial \Omega)$ to $L^{\infty}\left(\Omega_{d}\right)$, and the last equality follows since $\left.G^{x}\right|_{\partial \Omega} \in H^{s}(\partial \Omega)$.

Combining the formulas for the discrete and continuous problems, we have established the error representation

$$
\left(u-u_{h}\right)(x)=\left\langle G^{x}-G_{h}^{x}, f\right\rangle_{\partial \Omega} .
$$

Thus, the stated error estimates will be proved if we can show the following proposition.

Proposition 1. For $x \in \Omega_{d}$ the following bounds hold:

$$
\left\|G^{x}-G_{h}^{x}\right\|_{L^{\infty}(\partial \Omega)} \leq C_{d, r} \ell_{h, r} h^{r}
$$

and

$$
\left\|G^{x}-G_{h}^{x}\right\|_{W_{\infty}^{1}(\partial \Omega)} \leq C_{d, r} \ell_{h, r} h^{r-1} .
$$

Proof. Let $B$ be a ball of radius $d / 2$ centered at $x$. For this choice of $B$, the precise statement of the $L^{\infty}$ localization estimates given in equation (4) in the introduction is

$$
\left\|u-u_{h}\right\|_{L^{\infty}(B / 2)} \leq C\left\{\ell_{h, r} \min _{\chi \in S^{h}}\|u-\chi\|_{L^{\infty}(B)}+d^{-N / 2-s}\left\|u-u_{h}\right\|_{H^{-s}(B)}\right\},
$$

where $B / 2$ is a ball concentric with $B$ and of half the radius, [13. Using this and the symmetry of the discrete and continuous Green's functions, we find, for fixed $y \in \partial \Omega$,

$$
\begin{array}{r}
\left|G^{x}(y)-G_{h}^{x}(y)\right|=\left|G^{y}(x)-G_{h}^{y}(x)\right| \leq C\left\{\ell_{h, r} \min _{\chi \in S^{h}}\left\|G^{y}-\chi\right\|_{L^{\infty}(B)}\right. \\
\left.+d^{-N / 2-s}\left\|G^{y}-G_{h}^{y}\right\|_{H^{-s}(B)}\right\}=I+I I .
\end{array}
$$

Beginning with $I$, we recall the well known regularity result for the Green's function [10],

$$
\left|D_{x}^{\alpha} G^{y}(x)\right| \leq C|x-y|^{2-N-|\alpha|},
$$

where $C$ depends on $|\alpha|, A$, and $\Omega$. Using this and the approximation properties of the subspace, we see that

$$
\begin{aligned}
I & \leq C \ell_{h, r} h^{r}\left\|G^{y}\right\|_{W_{\infty}^{r}(B)} \leq C \ell_{h, r} h^{r} d(B, y)^{2-N-r} \\
& \leq C \ell_{h, r} h^{r}(d / 2)^{2-N-r} \leq C_{d, r} \ell_{h, r} h^{r} .
\end{aligned}
$$

For $I I$, we proceed by a duality argument. We have the bound

$$
\left\|G^{y}-G_{h}^{y}\right\|_{H^{-s}(B)} \leq\left\|G^{y}-G_{h}^{y}\right\|_{H^{-s}(\Omega)}=\sup _{\|\phi\|_{H^{s}=1}}\left(G^{y}-G_{h}^{y}, \phi\right) .
$$


For each fixed $\phi$, we let $w$ solve the dual problem, $A(v, w)=(v, \phi), \quad \forall v \in H^{1}(\Omega)$. It then follows that, letting $w_{h}$ satisfy $A\left(w-w_{h}, v\right)=0, \forall v \in S_{r}^{h}$, and using, for instance, [14] for $L^{\infty}$ estimates up to the boundary,

$$
\begin{aligned}
\left|\left(G^{y}-G_{h}^{y}, \phi\right)\right| & =\left|A\left(G^{y}-G_{h}^{y}, w\right)\right|=\left|A\left(G^{y}, w-w_{h}\right)\right|=\left|w-w_{h}(y)\right| \\
& \leq\left\|w-w_{h}\right\|_{L^{\infty}} \leq C \ell_{h, r} h^{r}\|w\|_{W_{\infty}^{r}(\Omega)} .
\end{aligned}
$$

Then, using Morrey's inequality (see [7, p. 266) followed by a Sobolev inequality and elliptic regularity, we estimate

$$
\begin{aligned}
\|w\|_{W_{\infty}^{r}} & \leq C \epsilon^{\frac{1-N-\epsilon}{N+\epsilon}}\|w\|_{W_{N+\epsilon}^{r+1}} \leq C \epsilon^{-1}\|w\|_{W_{N+\epsilon}^{r+1}} \leq C \epsilon^{-1}\|w\|_{H^{r+1+\frac{N+\epsilon-2}{2+\epsilon / N}}} \\
& \leq C \epsilon^{-1}\|w\|_{H^{r+N / 2+\epsilon / 2}} \leq C \epsilon^{-1}\|\phi\|_{H^{r+N / 2-2+\epsilon / 2}} .
\end{aligned}
$$

Hence, if we take $s=r+N / 2-2+\epsilon / 2$ in expression $I I$, we get

$$
I I \leq C d^{-N-r+2-\epsilon / 2}\left\|G^{y}-G_{h}^{y}\right\|_{H^{-r-N / 2+2-\epsilon / 2}(\Omega)} \leq C \epsilon^{-1} d^{-N-r+2-\epsilon / 2} \ell_{h, r} h^{r}
$$

and by choosing $\epsilon=-2(\log d)^{-1}$, we obtain

$$
C \epsilon^{-1} d^{-N-r+2-\epsilon / 2}=-C(\log d) d^{-N-r+2} d^{(\log d)^{-1}}=C_{d, r} .
$$

This proves (10).

For (11), we use inverse estimates. Taking an arbitrary element, $\tau$, abutting the boundary, and noting that the estimates in part 1 apply to the entire element, we have, for an appropriately chosen interpolant, $G_{I}^{x}$,

$$
\begin{aligned}
\left\|G^{x}-G_{h}^{x}\right\|_{W_{\infty}^{1}(\tau)} & \leq\left\|G^{x}-G_{I}^{x}\right\|_{W_{\infty}^{1}(\tau)}+\left\|G_{I}^{x}-G_{h}^{x}\right\|_{W_{\infty}^{1}(\tau)} \\
& \leq\left\|G^{x}-G_{I}^{x}\right\|_{W_{\infty}^{1}(\tau)}+C h^{-1}\left\|G_{I}^{x}-G_{h}^{x}\right\|_{L^{\infty}(\tau)} \\
& \leq C h^{r-1} d^{-N-r+1}+C h^{-1}\left\|G^{x}-G_{h}^{x}\right\|_{L_{\infty}(\tau)} \leq C_{d, r} h^{r-1} .
\end{aligned}
$$

This estimate holds for all elements abutting the boundary. Restricting to the boundary, we obtain the desired estimate.

2.2. Proof of sharpness. We next show that the order of convergence given in Theorem 1 is sharp, modulo the logarithmic factor. We will restrict ourselves to the piecewise linear case $(r=2)$, but the proof can be generalized to higher order elements. The basic idea is simple: If we take our boundary data, $f$, to be $\delta_{\tau}\left(y_{0}\right)$, the tangential derivative of the delta function centered at $y_{0} \in \partial \Omega$, then we can move $y_{0}$ along the boundary within a fixed element without changing $\langle v, f\rangle_{\partial \Omega}$ for $v \in S^{h}$, and hence without changing $u_{h}$. The true solution, $u$, however, will change by $O(h)$ over a significant portion of the domain, so the order of convergence can be no greater than 1 . The following formalizes this argument.

Proposition 2. There exists a domain, $\Omega$, an interior subdomain $\Omega_{0}$ and a family of finite element subspaces satisfying the hypotheses of Theorem 1 with $r=2$, such that the following holds. For $\alpha>1$ there exists no constant, $C$, such that $\left\|u-u_{h}\right\|_{L_{\infty}\left(\Omega_{0}\right)}<C h^{\alpha}\|f\|_{W_{1}^{-1}}$ for all $f \in W_{1}^{-1}(\partial \Omega)$.

Proof. We begin by choosing a smooth domain, $\Omega \in R^{2}$, such that $\partial \Omega$ contains an interval, $I=[-a, a] \in R_{x}$ where $R_{x}$ denotes the $x$-axis, and fixing $A(u, v)=$ $(\nabla u, \nabla v)+(u, v)$. We note that it is possible to choose some disk, $D \subset \Omega$, of positive radius, and some $[-b, b] \subset I$ such that for $\forall x \in D$ and $\forall y \in[-b, b], G_{\tau \tau}^{x}(y) \leq \kappa<0$, where $G_{\tau \tau}^{x}$ is the second tangential derivative of the Green's function. To see this, 
we let $G_{1}^{x}(y)$ be the Green's function on $R^{2}$ for $-\Delta u+u$ and let $G_{2}^{x}=G_{1}^{x}+G_{1}^{\bar{x}}$, where $\bar{x}$ is $x$ reflected across $R_{x}$. Since $\frac{\partial}{\partial n} G_{2}^{x}=0$ on $[-a, a]$, we can write

$$
G^{x}=G_{2}^{x}(y)+\rho^{x}
$$

where $\rho^{x}$ is a correction term with all derivatives uniformly bounded independent of $x$, for $x$ bounded away from $\partial \Omega / I$. Since $G_{2, \tau \tau}^{x}(t, 0) \rightarrow-\infty$ as $x \rightarrow(t, 0)$, it follows that we can choose $D$ and $[-b, b]$ with the property stated.

Next we fix $h<2 b$ and construct two boundary data functions as follows. Let $\delta_{1}^{h}$ be a smooth positive function with support in $(-b,-b+h / 4)$ such that $\left\|\delta_{1}^{h}\right\|_{L^{1}}=1$ and let $\delta_{2}^{h}$ be given by $\delta_{2}^{h}(y)=\delta_{1}^{h}(y-h / 2)$. We define $f_{i}^{h}=\left(-\delta_{i}^{h}\right)^{\prime}$ and note that for smooth $\phi$,

$$
\left\langle f_{1}^{h}, \phi\right\rangle=\left\langle\delta_{1}^{h}, \phi^{\prime}\right\rangle=\phi^{\prime}(y)
$$

for some $y=y(\phi) \in(-b,-b+h / 4)$, and analogously for $f_{2}^{h}$. Similarly, we obtain

$$
\left\|f_{i}^{h}\right\|_{W_{1}^{-1}}=\sup _{\|v\|_{W_{\infty}^{1}}=1}\left\langle f_{i}^{h}, v\right\rangle=\sup _{\|v\|_{W_{\infty}^{1}}=1}\left\langle\delta_{i}^{h}, v^{\prime}\right\rangle \leq \sup _{\|v\|_{W_{\infty}^{1}=1}^{1}}\left\|\delta_{i}^{h}\right\|_{L^{1}}\left\|v^{\prime}\right\|_{L^{\infty}} \leq 1 .
$$

We let $u_{i}$ be the solution to the continuous problems with boundary data $f_{i}$, and note that for $x \in D, \exists y_{1} \in[-b,-b+h / 4]$ and $y_{2} \in[-b+3 h / 4,-b+h]$ such that

$$
\begin{aligned}
u_{1}(x) & =\left\langle G^{x}, f_{1}^{h}\right\rangle_{\partial \Omega}=G_{\tau}^{x}\left(y_{1}\right)>G_{\tau}^{x}\left(y_{2}\right)+\kappa h / 2 \\
& =\left\langle G^{x}, f_{2}^{h}\right\rangle_{\partial \Omega}+\kappa h / 2=u_{2}(x)+\kappa h / 2 .
\end{aligned}
$$

Now, we construct a triangulation of size $h,\left\{\tau_{i}^{h}\right\}$, on $\Omega$ such that $[-b,-b+h] \in$ $\left.\left\{\tau_{i}^{h}\right\}\right|_{\partial \Omega}$ and we let $S_{r}^{h}$ be the space of piecewise linear elements on $\left\{\tau_{i}^{h}\right\}$. We have $\left\langle v, f_{1}^{h}\right\rangle_{\partial \Omega}=\left\langle v, f_{2}^{h}\right\rangle_{\partial \Omega} \forall v \in S_{r}^{h}$, so $u_{1, h}=u_{2, h}$. From this and the above, we find that for $x \in D,\left|u_{i}-u_{i, h}(x)\right|>\kappa h / 4$ for either $i=1$ or 2 . Since $\kappa$ is independent of $h$ and $\left\|f_{i}^{h}\right\|_{W_{1}^{-1}}$ is bounded independent of $h$, this proves the proposition.

Remark 1 . Since the subdomain, $D$, in the proof of the proposition is also independent of $h$, it follows that $\left\|u_{1}-u_{1, h}\right\|_{L^{p}(D)}+\left\|u_{2}-u_{2, h}\right\|_{L^{p}(D)} \geq\left\|u^{1}-u^{2}\right\|_{L^{p}(D)} \geq$ $C(p) \kappa h$ for any $1 \leq p \leq \infty$, so the interior order of convergence can be no greater than 1 in any $L^{p}$ norm.

Remark 2. It can also be seen that the powers of $d$ in Theorem 1 are sharp, modulo the factor of $\log (1 / d)$. If we take $f=\delta_{y}$ for $y \in \partial \Omega$, then the exact solution is $G^{y}$, and hence by the decay properties of the Green's function and the approximation properties of the subspace, we see that $\left\|G^{y}-G_{h}^{y}\right\|_{L^{\infty}\left(\Omega_{d}\right)} \geq C h^{r} d^{-N-r+2}$.

\section{Smoothing}

We have seen above that for very low regularity boundary data it is not always possible to define the finite element solution to problem (1), and that even when the finite element solution is well defined, the interior rate of convergence is sometimes suboptimal. In this section we show how smoothing the boundary data allows one to use the standard finite element method on problems with data in any negative norm space, and also provides a means for improving suboptimal interior rates of convergence.

We begin by assuming we have a family of convolution kernels, $K_{H, s}^{m}$ which have smoothing degree $s$,

$$
\left\|K_{H, s}^{m} * f\right\|_{W_{p}^{k}} \leq C H^{l-k}\|f\|_{W_{p}^{l}}, \quad \forall p, \quad \text { and } \quad 0 \leq l \leq k \leq l+s
$$


and approximation degree $m$,

$$
\left\|f-K_{H, s}^{m} * f\right\|_{W_{p}^{k}} \leq C H^{l-k}\|f\|_{W_{p}^{l}}, \quad \forall p, \quad \text { and } \quad 0 \leq k \leq l \leq m+l .
$$

One family satisfying these on $R^{N}$ is defined in [5], and we refer the reader there for more details. Here we only note that it is possible to choose $K_{H, s}^{m}$ to be piecewise polynomial with support proportional to $H$. From (12) and (13), and from $\left(K_{H, m}^{s} * f, \phi\right)=\left(f, K_{H, m}^{s} * \phi\right)$, we obtain the smoothing property,

$$
\left\|K_{H, s}^{m} * f\right\|_{W_{p}^{-l}} \leq C H^{l-k}\|f\|_{W_{p}^{-k}}, \quad \forall p, \text { and } 0 \leq l \leq k \leq l+s
$$

and the approximation property,

$$
\left\|f-K_{H, s}^{m} * f\right\|_{W_{p}^{-l}} \leq C H^{l-k}\|f\|_{W_{p}^{-k}}, \quad \forall p, \text { and } 0 \leq k \leq l \leq m+l .
$$

Fixing $H, s$ and $m$, we define $\tilde{u}_{h} \in S_{r}^{h}$ by the relationship

$$
A\left(\tilde{u}_{h}, v\right)=\left\langle K_{H, s}^{m} * f, v\right\rangle_{\partial \Omega} .
$$

Note that this definition makes sense for $f \in W_{p}^{-k}(\partial \Omega)$ as long as $k \leq s+1$, so we have succeeded in defining a finite element approximation to problem (1) for data in any negative norm space. The main theorem in this section shows how the rate of convergence of this approximation depends on the choice of $H, m$ and $s$.

Theorem 2. Let $u$ be the solution to problem (1), and let $\tilde{u}_{h}$ be as defined by equation (16). If the family of smoothing kernels, $\left\{K_{H, s}^{m}\right\}$, satisfies (14) and (15) and if we choose $H=h^{\frac{r}{m+k}}$, then

$$
\left\|u-\tilde{u}_{h}\right\|_{L^{\infty}\left(\Omega_{d}\right)} \leq C(d) \ell_{h, r} h^{r\left(\frac{m}{m+k}\right)}\|f\|_{W_{1}^{-k}}, \forall 0 \leq k \leq s
$$

where $C(d)=C \sup \left\{C_{d, r}, d^{2-N-m-k}\right\}$ and $m$ and $s$ are, respectively, the approximation and smoothing degrees of the kernel.

Before giving the proof of this theorem, we take a moment to comment on the form of this estimate. First, as a point of reference, note that for $k=0$, (17) reduces to (8) with $l=0$. It is also possible to prove an estimate analogous to (17) which corresponds to (8) with $l=1$, but (17) is the better of the two for almost all values of $r, m$ and $k$ that are likely to appear in practice, so we omit the second estimate here. Second we note that this theorem implies that by sufficiently smoothing the boundary data it is possible to obtain interior convergence rates as close to optimal as one likes. However, there is a trade-off. To obtain (17) we must choose $H=h^{\frac{r}{m+k}}$ so, as $m$ increases, the support of the kernel grows, which increases the cost of computing the convolution in (16) and also degrades the approximation near the boundary. This second effect is manifested in the additional factor of $d^{r-m-k}$ appearing in the estimate when $m+k>r$. We now move on to the proof.

Proof of Theorem 2. Let $\tilde{u}$ solve problem (1), with $f$ replaced by $K_{H, s}^{m} * f$. Then we have

$$
\left\|u-\tilde{u}_{h}\right\|_{L^{\infty}\left(\Omega_{d}\right)} \leq\|u-\tilde{u}\|_{L^{\infty}\left(\Omega_{d}\right)}+\left\|\tilde{u}-\tilde{u}_{h}\right\|_{L^{\infty}\left(\Omega_{d}\right)}=I+I I .
$$


Using the Green's function representation for the solution of the continuous problem, we have, for $x \in \Omega_{d}$,

$$
\begin{aligned}
I & =|u-\tilde{u}(x)|=\left|\left\langle G^{x}, f-K_{H, s}^{m} * f\right\rangle_{\partial \Omega}\right| \\
& \leq\left\|G^{x}\right\|_{W_{\infty}^{m+k}(\partial \Omega)}\left\|f-K_{H, s}^{m} * f\right\|_{W_{1}^{-m-k}} \\
& \leq C d^{2-N-m-k} H^{m}\|f\|_{W_{1}^{-k}} .
\end{aligned}
$$

For $k \leq s$, Theorem 1 gives

$$
I I \leq C_{d, r} \ell_{h, r} h^{r}\left\|K_{H, s}^{m} * f\right\|_{L^{1}} \leq C_{d, r} \ell_{h, r} h^{r} H^{-k}\|f\|_{W_{1}^{-k}} .
$$

Putting this all together, and choosing $H=h^{\frac{r}{m+k}}$ gives

$$
\begin{aligned}
\left\|u-\tilde{u}_{h}\right\|_{L^{\infty}\left(\Omega_{d}\right)} & \leq C\left(d^{2-N-m-k} H^{m}+C_{d, r} \ell_{h, r} h^{r} H^{-k}\right)\|f\|_{W_{1}^{-k}} \\
& \leq C(d) \ell_{h, r} h^{r\left(\frac{m}{m+k}\right)}\|f\|_{W_{1}^{-k}} .
\end{aligned}
$$

In the next section we will see how smoothing can be applied to problems with concentrated boundary data. Here we give a sketch of a potential application to a problem where the data contains high frequency oscillations. By this we mean that the boundary function, $f(y)$, can be decomposed into a regular component, $f_{r}(y)$, and an oscillating component, $f_{o}(y)$, such that for some $\lambda>>1$,

$$
\left\|f_{r}\right\|_{W_{1}^{-k}} \leq 1 \text { and }\left\|f_{o}\right\|_{W_{1}^{-k}} \leq C \lambda^{-k} .
$$

One situation where this could occur is in the solution of forward problems or inverse problems based on experimental data with certain types of noise. With this type of problem, it is natural to ask how the oscillations will affect the performance of the method, and what type of preliminary smoothing of the data might be beneficial. For this, we use (17) and find that for the appropriate choice of $H$,

$$
\left\|u-\tilde{u}_{h}\right\|_{L^{\infty}\left(\Omega_{d}\right)} \leq C(d) \ell_{h, r} h^{r\left(\frac{m}{m+k}\right)}\|f\|_{W_{1}^{-k}} \leq C(d) \ell_{h, r} h^{r\left(\frac{m}{m+k}\right)}\left(1+C \lambda^{-k}\right) .
$$

For fixed $r, m$ and $h$, we can easily minimize this with respect to $k$ and choose the corresponding kernel. In particular, we note that this analysis suggests that smoothing is most beneficial if the $L^{1}$ norm of the oscillating component is significantly larger than that of the regular component.

\section{NUMERICAL ILLUSTRATIONS}

In this section we present some numerical results for problem (1) with $A=-\Delta$ and $\Omega$ the disk of radius 1 . We note that this choice of $A$ is not coercive over $H^{1}$, and so does not satisfy all of the hypotheses required for our proofs. However, we will see that we nevertheless obtain the predicted rates of convergence, suggesting that our results are robust.

We give results for the following three test problems. Parameterizing the boundary by arc length, we define boundary functions and corresponding solutions

$$
\begin{aligned}
f_{1}=-2 \pi \delta_{0}+1, & u_{1}=\log \left[(x-1)^{2}+y^{2}\right], \\
f_{2}=2 \pi \delta_{0}^{\prime}, & u_{2}=\frac{2 y}{(x-1)^{2}+y^{2}}, \\
f_{3}=2 \pi \delta_{0}^{\prime \prime}, & u_{3}=\frac{2 x\left(x^{2}+y^{2}+1\right)-4\left(x^{2}+y^{2}\right)}{\left(y^{2}+(x-1)^{2}\right)^{2}}
\end{aligned}
$$


where $\delta_{0}, \delta_{0}^{\prime}$, and $\delta_{0}^{\prime \prime}$ are the delta functions centered at $\theta=0$, and the first and second derivatives of the delta function centered at $\theta=0$, respectively.

We describe briefly the derivation of these exact solutions. For $u_{1}$, we begin with the solution and compute $f_{1}=\left(u_{1}\right)_{n}$ directly. For $u_{2}$ and $u_{3}$, this becomes more complicated, so instead we begin with the boundary functions, $f_{2}$ and $f_{3}$, and compute the solutions, $u_{2}$ and $u_{3}$, using the modified Green's function representation,

$$
u(y)-u(0)=\left\langle\tilde{G}^{y}, f\right\rangle_{\partial \Omega},
$$

where

$$
\tilde{G}^{y}(x)=\frac{-1}{2 \pi}\left(\log |x-y|+\log \left|\frac{x}{|x|^{2}}-y\right|\right) .
$$

Note that these solutions are only determined up to an additive constant. For the first two problems we select the solution with mean zero. For the third problem the solution is not in $L^{1}$, and so computing the mean becomes a delicate issue which we choose to circumnavigate by taking instead the solution which is zero at $(x, y)=(0,0)$. In our numerical implementation, we compute the mean zero approximation for all three problems, and then shift the approximation to the third problem to set it equal to zero at $(x, y)=(0,0)$.

Next we give more detail on our numerical implementation. All computations were performed on quasi-uniform meshes using pie-shaped elements to fit the boundary exactly. These meshes were chosen to be uniform over the set, $D=\{(x, y),|x|+|y| \leq 1 / 2\}$, which is the subdomain over which we report errors. The stiffness matrix was computed exactly, using elementary geometric considerations for elements abutting the boundary. The $L^{2}$ inner product matrix used in the mean zero computations was again computed exactly for elements not abutting the boundary. For elements abutting the boundary, the portion of the integral over the "skin" layer was computed in polar coordinates using 3-point quadrature for the " $r$ " integral followed by 8-point quadrature for the " $\theta$ " integral. Boundary integrals were divided into singular and nonsingular parts. The nonsingular part of each integral was computed by breaking the integral into segments where the integrand was smooth and using 8-point quadrature on each segment. The singular part was computed exactly, using the exact expression for the values of the basis functions on the boundary.

There are two points here which require additional comment. First, note that $\delta^{\prime}$ is not quite in $W_{1}^{-1}$. From the point of view of rates of convergence, it is close enough, since it may be thought of as the limit of a sequence of smoothed approximations with bounded $W_{1}^{-1}$ norm, as in the proof of Theorem 2 above. However, from a computational standpoint this noninclusion presents a small problem, since $\left\langle\delta_{x_{0}}^{\prime}, v\right\rangle$ is not defined for $v \in S^{h}$ and $x_{0}$ a mesh point. In our computations we avoid this issue by being careful not to place mesh points on this singularity. Second, we note that $\left\langle\delta_{x_{0}}^{\prime \prime}, v\right\rangle$ is (nearly) zero for all $v \in S^{h}$, since we are only considering piecewise linear elements $\left(\left\langle\delta_{x_{0}}^{\prime \prime}, v\right\rangle\right.$ would be exactly zero for linear elements on a polygonal domain). Thus, for this function with unsmoothed data, the finite element solution will just be (nearly) zero for all $h$, and so we do not include this case in our computations.

Our results are summarized in the tables below. The first table contains errors and observed rates of convergence for the three test problems for $h=1 / 32,1 / 64$ and $1 / 128$ for several choices of smoothing kernels and mesh types (The value of $H$ used for each function and smoothing kernel is based on the theory from 
TABLE 1. Errors and Observed Rates of Convergence (For $H$, see

Table 2.)

\begin{tabular}{|lc|cc|cc|cc|}
\hline kernel/mesh & $\mathrm{h}$ & $\delta$ & & $\delta^{\prime}$ & & $\delta^{\prime \prime}$ & \\
\hline \multirow{3}{*}{ none/regular } & $\frac{1}{32}$ & 5.46 & - & 26.7 & - & - & - \\
& $\frac{1}{64}$ & 1.36 & 2.00 & 13.1 & 1.02 & - & - \\
& $\frac{1}{128}$ & 0.34 & 2.00 & 6.1 & 1.10 & - & - \\
$K_{H, 2}^{4} /$ regular & $\frac{1}{32}$ & 5.53 & - & 2.25 & - & 4.11 & - \\
& $\frac{1}{64}$ & 1.39 & 2.00 & 0.55 & 2.05 & 1.95 & 1.13 \\
& $\frac{1}{128}$ & 0.35 & 2.00 & 0.13 & 2.07 & 0.88 & 1.20 \\
& & & & & & \\
$K_{H, 2}^{2} /$ distorted & $\frac{1}{32}$ & 13.1 & - & 4.49 & - & 7.28 & - \\
& $\frac{1}{64}$ & 3.8 & 1.78 & 1.92 & 1.23 & 4.20 & 0.94 \\
& $\frac{1}{128}$ & 1.1 & 1.82 & 0.81 & 1.24 & 1.96 & 0.96 \\
$K_{H, 2}^{4} /$ distorted & $\frac{1}{32}$ & 12.7 & - & 3.28 & - & 6.15 & - \\
& $\frac{1}{64}$ & 3.6 & 1.84 & 1.05 & 1.64 & 2.58 & 1.24 \\
& $\frac{1}{128}$ & 1.0 & 1.88 & 0.34 & 1.65 & 1.07 & 1.28 \\
\hline
\end{tabular}

Section 3, and can be found by referring to the corresponding entry in Table 2). The first two entries are for computations on a very regular family of meshes, chosen so the diameter of the elements varied by no more than a factor of $\sqrt{2}$ over the whole domain. The first entry is for unsmoothed data and the second for data smoothed with a kernel of approximation degree 4 . The second two entries are for a family of meshes distorted in such a way that the elements to one side of the singularity had diameter about 4 times that of the elements to the other side. On this mesh we give results for two choices of smoothing kernel, the first with approximation degree 2 and the second with degree 4 . We explain our reason for including results for this distorted family of meshes below, and for now only note that it still fits our definition of quasi-uniform. We also mention, for clarity, that the smoothing kernels depend on the mesh only through the global parameter, $h$, and thus were unaltered by the distortion of the mesh.

From this table, we see that for $u_{2}$ and $u_{3}$ there is a clear improvement both in the rate of convergence and in the actual size of the error when the data is smoothed (as noted above, one can expect no convergence at all for $u_{3}$ without smoothing). For $u_{1}$, on the other hand, the rate of convergence and the size of the error for smoothed data are basically the same as for unsmoothed data. This further supports the conclusion drawn from the predicted rates of convergence that smoothing is most beneficial when the data has regularity lower than $L^{1}$.

In the second table, we compare the results in the first table to the predictions given by our theory. For each boundary function, we list the size of the support of our chosen smoothing kernel, the predicted rate of convergence, and the observed rate of convergence, averaged over $h=1 / 32$ to $h=1 / 128$. We note that $f_{1}, f_{2}$, and $f_{3}$ are almost in $L^{1}, W_{1}^{-1}$ and $W_{1}^{-2}$ respectively, and base our choice of kernel 
TABle 2. Predicted and Observed Rates of Convergence

\begin{tabular}{|l|ccc|ccc|ccc|}
\hline kernel/mesh & \multicolumn{3}{|c|}{$\delta$} & \multicolumn{3}{c|}{$\delta^{\prime}$} & \multicolumn{3}{|c|}{$\delta^{\prime \prime}$} \\
\hline & $\mathrm{H}$ & pred. & obs. & $\mathrm{H}$ & pred. & obs. & $\mathrm{H}$ & pred. & obs. \\
none/regular & - & 2 & 2.00 & - & 1 & 1.06 & - & - & - \\
$K_{H, 2}^{4} /$ regular & $h^{1 / 2}$ & 2 & 2.00 & $h^{2 / 5}$ & $8 / 5$ & 2.06 & $h^{1 / 3}$ & $4 / 3$ & 1.17 \\
$K_{H, 2}^{2} /$ distorted & $h$ & 2 & 1.80 & $h^{2 / 3}$ & $4 / 3$ & 1.23 & $h^{1 / 2}$ & 1 & 0.94 \\
$K_{H, 2}^{4} /$ distorted & $h^{1 / 2}$ & 2 & 1.86 & $h^{2 / 5}$ & $8 / 5$ & 1.65 & $h^{1 / 3}$ & $4 / 3$ & 1.26 \\
\hline
\end{tabular}

and our predicted rates of convergence on these near inclusions. For unsmoothed data on a regular mesh, we obtain the predicted rate of convergence for $u_{1}$ and $u_{2}$. For smoothed data on a regular mesh, we again obtain the predicted rate for $u_{1}$, but for $u_{2}$, we obtain optimal order convergence, which is not predicted by the theory. An informal explanation of this unexpectedly high convergence rate can be given by returning to the error representation formula derived in Section 2, equation (9), transferring the smoothing kernel from $\delta^{\prime}$ to $G_{h}^{x}$, and then applying superconvergence results for gradient recovery, to $\left|\nabla G-\nabla K_{H, 2}^{4} * G_{h}^{x}\right|$. We will not pursue this further here, but choose instead to give results for the distorted mesh described above where this superconvergence phenomenon should no longer occur. These results are given in the last two lines of the table, and we see here that the rates follow the predicted pattern, although they are slightly depressed, owing to the distortion of the mesh.

\section{REFERENCES}

1. Ivo Babuška, Joseph E. Flaherty, William D. Henshaw, John E. Hopcroft, Joseph E. Oliger, and Tayfun Tezduyar (eds.), Modeling, Mesh Generation, and Adaptive Numerical Methods for Partial Differential Equations, The IMA Volumes in Mathematics and its Applications, vol. 75, New York, Springer-Verlag, 1995. MR.1370242 (96g:65002)

2. Ivo Babuška and Victor Nistor, Boundary value problems in spaces of distributions on smooth and polygonal domains, preprint.

3. - Interior numerical approximation of boundary value problems with a distributional data, Numer. Methods Partial Differential Equations 22 (2006), no. 1, 79-113. MR 2185526 (2006m:65253)

4. Christine Bernardi, Optimal finite-element interpolation on curved domains, SIAM J. Numer. Anal. 26 (1989), no. 5, 1212-1240. MR1014883 (91a:65228)

5. J. H. Bramble and A. H. Schatz, Higher order local accuracy by averaging in the finite element method, Math. Comp. 31 (1977), no. 137, 94-111. MR0431744 (55:4739)

6. James H. Bramble and J. Thomas King, A robust finite element method for nonhomogeneous Dirichlet problems in domains with curved boundaries, Math. Comp. 63 (1994), no. 207, 1-17. MR 1242055 (94i:65112)

7. Lawrence C. Evans, Partial Differential Equations, Graduate Studies in Mathematics, vol. 19, American Mathematical Society, Providence, RI, 1998. MR1625845 (99e:35001)

8. George J. Fix, Max D. Gunzburger, and Janet S. Peterson, On finite element approximations of problems having inhomogeneous essential boundary conditions, Comput. Math. Appl. 9 (1983), no. 5, 687-700. MR726817 (85b:65102)

9. Donald A. French and J. Thomas King, Approximation of an elliptic control problem by the finite element method, Numer. Funct. Anal. Optim. 12 (1991), no. 3-4, 299-314. MR.1143001 (92m:65144)

10. Ju. P. Krasovskiı̌, Properties of Green's functions, and generalized solutions of elliptic boundary value problems, Dokl. Akad. Nauk SSSR 184 (1969), 270-273. MR0237956 (38:6233)

11. J.-L. Lions and E. Magenes, Non-homogeneous Boundary Value Problems and Applications. Vol. I, Springer-Verlag, New York, 1972. MR0350177 (50:2670) 
12. Joachim A. Nitsche and Alfred H. Schatz, Interior estimates for Ritz-Galerkin methods, Math. Comp. 28 (1974), 937-958. MR0373325 (51:9525)

13. A. H. Schatz and L. B. Wahlbin, Interior maximum norm estimates for finite element methods, Math. Comp. 31 (1977), no. 138, 414-442. MR0431753 (55:4748)

14. Alfred H. Schatz, Pointwise error estimates and asymptotic error expansion inequalities for the finite element method on irregular grids. I. Global estimates, Math. Comp. 67 (1998), no. 223, 877-899. MR1464148 (98j:65082)

15. Lars B. Wahlbin, Local behavior in finite element methods, Handbook of Numerical Analysis, Vol. II, North-Holland, Amsterdam, 1991, pp. 353-522. MR.1115238

Department of Mathematics, Cornell University, Ithaca, New York 14853

Current address: Susquehanna International Group, 401 City Line Avenue, Bala Cynwyd, Pennsylvania 19004

E-mail address: als54@cornell.edu 